\title{
Nodes and networks in the neural architecture for language: Broca's region and beyond Peter Hagoort
}

\begin{abstract}
Current views on the neurobiological underpinnings of language are discussed that deviate in a number of ways from the classical Wernicke-Lichtheim-Geschwind model. More areas than Broca's and Wernicke's region are involved in language. Moreover, a division along the axis of language production and language comprehension does not seem to be warranted. Instead, for central aspects of language processing neural infrastructure is shared between production and comprehension. Three different accounts of the role of Broca's area in language are discussed. Arguments are presented in favor of a dynamic network view, in which the functionality of a region is co-determined by the network of regions in which it is embedded at particular moments in time. Finally, core regions of language processing need to interact with other networks (e.g. the attentional networks and the ToM network) to establish full functionality of language and communication.
\end{abstract}

\begin{abstract}
Addresses
Donders Institute for Brain, Cognition and Behaviour, Max Planck Institute for Psycholinguistics, Nijmegen, The Netherlands
\end{abstract}

Corresponding author: Hagoort, Peter (peter.hagoort@donders.ru.nl)

Current Opinion in Neurobiology 2014, 28:136-141

This review comes from a themed issue on Communication and language

Edited by Michael Brainard and Tecumseh Fitch

http://dx.doi.org/10.1016/j.conb.2014.07.013

0959-4388/ (c) 2014 Published by Elsevier Ltd.

\section{Introduction}

Our capacity for language is deeply rooted in our biological make-up. We all share the capacity to acquire language within the first few years of life, without any formalized teaching programme. Despite its complexity we master our native language well before we can lace our shoes or perform simple calculations. This is all based on the universal availability of a language-ready brain. At the same time, few other cognitive systems in humans show as much variability as language. Language comes in very different surface forms, at most levels of organization. The more than 6000 different languages still in existence today vary widely in their sound repertoires, their grammatical structures, or the meaning that the lexical items code for. In addition to the variability in the world's languages, there is individual variation in language skills. Some people command only a limited vocabulary and simple sentence structures, whereas others are polyglots speaking multiple languages fluently, or can do simultaneous translation between languages. This variability is underpinned by differences at the genetic level and in the cultural trajectories that have shaped the linguistic phenotypes. Despite all these differences, they are variations on a theme. The theme is the neurobiological infrastructure that is largely shared among members of our species.

Although human language skills presumably have their precursors in the communication and cognitive systems of other species (cf. $\left[1^{\bullet}\right]$ ), the architecture of our language system sets us apart from other species and is uniquely human. It is characterized by a tripartite architecture [ $2^{\bullet \bullet}$ ] that enables us to map sound onto meaning (in listening) or meaning onto sound (in speaking). Next to sound and meaning, there is syntax, which enables the well-formed grouping of words into longer utterances. At a very general level, for all three information types (sound, syntax, meaning), one can make a distinction between two crucial components. The one relates to the common assumption that the basic building blocks of linguistic knowledge get encoded and consolidated in the course of language acquisition. This is what I hence refer to as the Memory component of the human language system, and is more usually called the mental lexicon in the field of psycholinguistics. Crucially, however, language processing is more than the retrieval of lexical knowledge and goes beyond the simple concatenation of retrieved lexical items. The expressive power of human language derives from the possibility to combine elements from memory in often novel ways. This creative aspect led Wilhelm von Humboldt [3] to characterize language as a system which makes infinite use of finite means. I have called this process of deriving new and complex meaning from the lexical building blocks Unification [4,5 ${ }^{\circ}$. Unification thus refers to the on-line assembly of lexical building blocks into larger structures, with contributions from context and general world knowledge. It instantiates what in linguistic theories is often called the compositionality of language. However this does not entail a commitment to any particular account of compositionality (cf. [6]). The cognitive infrastructure of Memory and Unification is supported by the neurobiological infrastructure of the human brain to which I now turn. 


\section{The neurobiology of language}

For more than a century the neurobiological model that has dominated the field was the Wernicke-LichtheimGeschwind (WLG) model (see Figure $\left.1 ; 7^{\bullet \bullet}\right]$ ). In this model, the human language faculty was situated in the left perisylvian cortex, with a strict division of labor between the frontal and temporal regions. Wernicke's area in left temporal cortex was assumed to subserve the comprehension of speech, whereas Broca's area in left inferior frontal cortex was claimed to subserve language production. The arcuate fasciculus connected these two areas.

Despite its impact until this very day (e.g. [8]), the classical model is not a fully adequate account of the neurobiology of language. Although Broca's area, Wernicke's area and adjacent cortex are core nodes in the language network, the distribution of labor between these regions is different than was claimed in the WLG model. Lesions in Broca's region are known to impair not only language production but also language comprehension [9], whereas lesions in Wernicke's region also affect language production. More recently, functional neuroimaging studies provided further evidence that the classical view on the role of these regions is no longer tenable. For example, central aspects of language production and comprehension are subserved by shared neural circuitry $\left[10,11^{\bullet}\right]$. Moreover, the classical model focused on single word processing, whereas a neurobiological account of

\section{Figure 1}

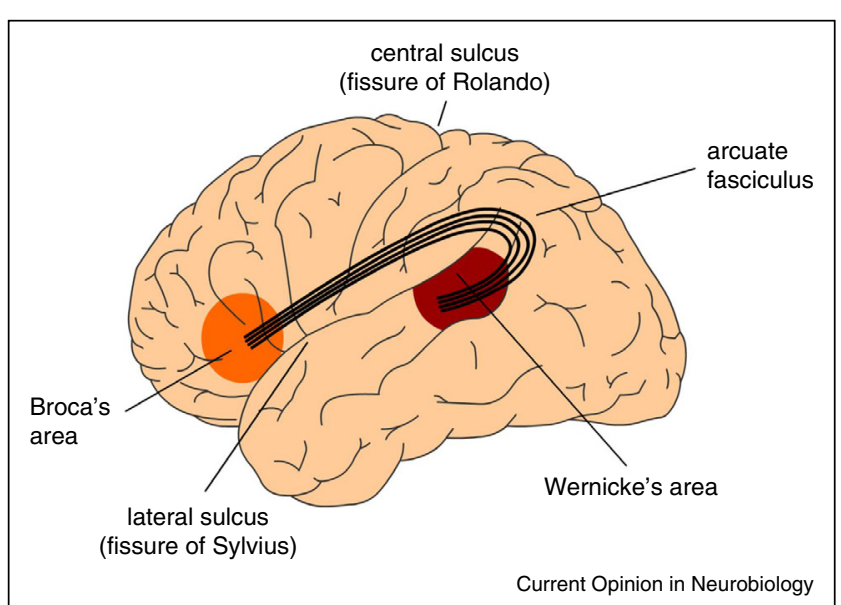

The classical Wernicke-Lichtheim-Geschwind model of the neurobiology of language. In this model Broca's area is crucial for language production, Wernicke's area subserves language comprehension, and the necessary information exchange between these areas (such as in reading aloud) is done via the arcuate fasciculus, a major fiber bundle connecting the language areas in temporal cortex (Wernicke's area) and frontal cortex (Broca's area). The language areas are bordering one of the major fissures in the brain, the so-called Sylvian fissure. Collectively, this part of the brain is referred to as perisylvian cortex. language processing in its full glory should also take into account what goes on beyond production and comprehension of single words. As a consequence of the mounting evidence against the classical WLG model, in recent years alternative neurobiological models for language have been proposed (e.g. $\left.\left[4,5^{\bullet}, 12,13^{\bullet}, 14-16\right]\right)$.

One very general alternative is the Memory, Unification, and Control (MUC) model $\left[4,5^{\circ}\right]$, in which the distribution of labor between Broca's area, Wernicke's area and adjacent cortical regions is as follows: regions in the temporal cortex and in the inferior parietal cortex (angular gyrus, supramarginal gyrus) subserve the knowledge representations that have been laid down in memory during acquisition. These regions store information including phonological word forms, morphological information, and the syntactic templates associated with noun, verbs, adjectives (for details, see $[4,17,18]$ ). They also include semantic convergence zones, but on the whole conceptual knowledge is quite widely distributed $\left[19^{\circ}\right]$. Dependent on knowledge type, different parts of temporal and parietal cortex are involved. Frontal regions (Broca's area and adjacent cortex) are crucial for unification operations. These operations generate larger structures from the building blocks that are retrieved from memory. Within left inferior frontal cortex (Unification Space), a spatial activation gradient is observed. The distribution of the activations seems to depend on the type of information that gets unified. Semantic unification recruits Brodmann's Area (BA) 47 and BA 45; syntactic unification has its focus in BA 45 and BA 44 [20 $0^{\circ}$, while phonological processes recruit BA 44 and ventral parts of BA 6. In addition, executive control needs to be exerted, such that the correct target language is selected, turn taking in conversation is orchestrated, the correct register is selected, attention is paid to the most relevant information in the input, and so forth. Control regions involve dorsolateral prefrontal cortex, and midline structure including the anterior cingulate cortex (ACG) and the parts of parietal cortex that are involved in attention [21]. In other models, the anterior temporal lobe (ATL) has been argued to be relevant for combinatorial operations [14,15]. One possibility is that this is limited to conceptual combinations for which the mapping of grammatical roles (e.g. subject, object) onto thematic roles (e.g. agent, patient) is not required [22]. In the latter case, the contribution of Broca's region is presumably highly relevant. Nevertheless, one has to realize that despite fairly general agreement on the organizational principles of the neural architecture for language, still much work needs to be done to establish the precise functional contributions of the nodes in this network.

The picture that I sketched is largely based on fMRI data on language processing that were acquired in the last two decades [23]. However, one limitation of fMRI and lesion based analyses is that it results in a much more static 
picture than is warranted by the highly dynamic organization of neural events that unfold in the language network. Such a picture favors accounts in which brain areas have fixed contributions independent of time and context. However, this static view is incorrect. Language functions do not reside in single brain regions. Instead, language is subserved by dynamic networks of brain regions, including the ones just outlined. Ultimately the mapping of a given language function onto the neural architecture of the brain appears to be in terms of a network of brain regions instantiating that particular language function $\left[24^{\bullet}, 25-28\right]$. Typically, each node in such a network will participate dynamically in other functional networks as well. This view does not deny that a specific region (e.g. part of Broca's area) has an identifiable role, but it is crucial to understand that the execution of this role depends on the interaction with other regions that are part of the relevant network [29]. In short, "the mapping between neurons and cognition relies less on what individual nodes can do and more on the topology of their connectivity."'([27]; p. 184). Next to focusing on regions of interest (ROIs), one might want to analyze networks of interest (NOIs; $\left[24^{\circ}\right]$ ). Hereafter I will discuss in more detail one region, well-known for its acclaimed role in language, and its connection with rest of the language network.

\section{Broca's area and adjacent cortex (Broca's region), and its connectivity}

If there is one area that has classically been associated with the language faculty, it is Broca's area. Despite some disagreement in the literature [30], most authors agree that Broca's area comprises Brodmann's Areas (BA) 44 and 45 of the left hemisphere [31]. In the classical textbooks these areas coincide at the macroscopic level with the pars opercularis (BA 44) and the pars triangularis (BA 45) of the third frontal convolution. However, cytoarchitectonic analysis [32] shows that areas 44 and 45 do not neatly coincide with the sulci that form their boundaries in macro-anatomical terms. In addition, areas 44 and 45 show a number of clear cytoarchitectonic differences, one of which is that 45 has a granular layer IV, whereas 44 is dysgranular. Recent more fine-grained analysis including not only cyto-architectonics but also receptor-architectonics suggests furthermore that BA 44 and 45 can be further subdivided into more than a dozen areas, if one takes the distribution of receptors of different neurotransmitters and modulators into consideration [31,33]. In addition, the connectivity with other brain regions also varies across Broca's area [34]. In other words, Broca's area is not a natural kind in neuroanatomical terms. Results from studies on the functional anatomy of language clearly show that adjacent areas such as BA 47 and the ventral part of BA 6 are also involved in language processing. Hence it is more appropriate to refer to this part of the left inferior frontal cortex as Broca's region.
Figure 2

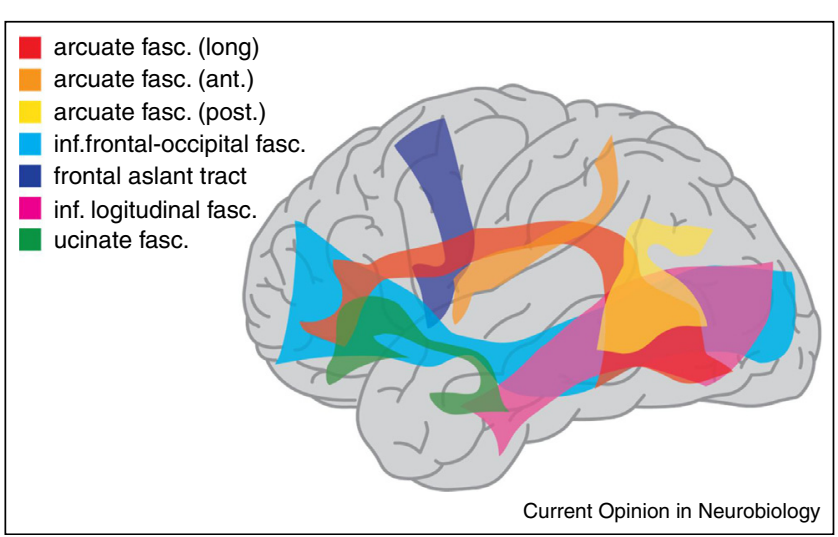

The language network visualized with diffusion tractography. In color, the major anatomical pathways connecting language relevant regions. After [34].

In the classical WLG model Broca's area is connected to Wernicke's area in temporal cortex. On the current view it is generally agreed that the connectivity profile between language-relevant regions in frontal, temporal and parietal cortex is much more extended. Figure 2 gives a schematic overview of the relevant fiber bundles that connect language-relevant brain regions based on diffusion tractography [34].

Another way to characterize connectivity is by means of resting state fMRI. Using this method, Xiang et al. [35] found a clear topographical functional connectivity pattern in the left inferior frontal, inferior parietal, and temporal regions (see Figure 3). In perisylvian cortex, patterns of functional connectivity obeyed the tripartite nature of language processing (phonology, syntax and semantics). These authors observed a topographical functional organization in the left perisylvian language network, in which areas are most strongly connected according to information type (i.e. phonological, syntactic, and semantic). Their results are consistent with the view that different parts of Broca's region are involved in, respectively, phonology, syntax, and semantics in connection with areas in inferior parietal and temporal cortex.

Not only the anatomical definition of Broca's region and its connectivity profile is more complex than classically assumed, also the functional role of Broca's region is still unresolved and hotly debated. In general terms, one can distinguish at least the following three accounts. One account attributes a very general role to prefrontal cortex including Broca's region. This domain-general role is that of selecting among competing sources of information, and cognitive control $\left[21,36^{\circ}, 37\right]$. According to this view, the activation of Broca's region is determined by general processing demands but not by the type of information 
Figure 3

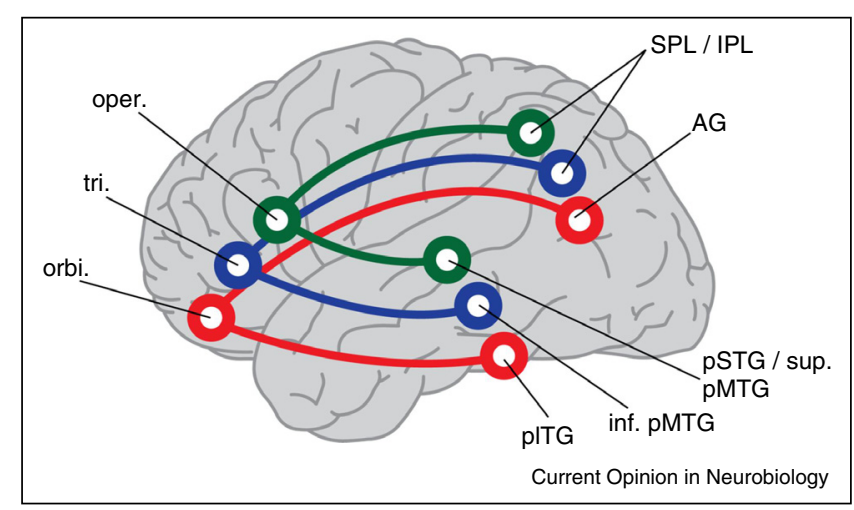

A schematic drawing of the topographical connectivity pattern between frontal and temporal/parietal cortex in the perisylvian language network, as revealed by resting state fMRI (after [35]). The strongest connections to the pars opercularis (oper.), part triangularis (tri.) and pars orbitalis (orbi.) of Broca's region are shown. SPL/IPL: superior parietal lobule/ inferior parietal lobule; AG: angular gyrus; PSTG: posterior superior temporal gyrus; sup. pMTG: superior posterior middle temporal gyrus; inf. pMTG: inferior posterior middle temporal gyrus; pITG: posterior inferior temporal gyrus.

that is being processed. This account is in strong contrast to the view that Broca's region has a special role in supporting the representations or the processing of complex syntactic structure [38,39], and that the anatomical and functional features of Broca's region in humans deviate from homologue areas in other primates, subserving a uniquely human capacity for syntax [40]. In this case at least part of Broca's region is considered to be language-specific [41]. A third position attributes both core language functions to part of Broca's region (though not necessarily specifically to syntax), as well a multiple demand system characteristics to other parts of Broca's region [42]. In the MUC framework [ $5^{\circ}$ ] Broca's region is not language-specific, but realizes its language-relevant unification function in connection to language-relevant areas in temporal and inferior parietal cortex that support the storage of different types of linguistic knowledge [43 ${ }^{\circ}$. Recently, a similar network view has been advocated by Fedorenko and Thompson-Schill [24 $\left.4^{\circ}\right]$. Overall, the currently dominating view is that the role of Broca's region and adjacent cortex should be characterized in more general terms than hierarchical or even sentencelevel processing. Certainly Broca's region as a whole is not an exclusive syntax area. To what extent a sub-parcellation of Broca's region [31,33] might reveal some regions to be more specific for language processing than others [42] remains to be seen.

Importantly, Broca's region is also involved in unification operations at the word level, as in morphological (de)composition [44-46]. Compositional and decompositional operations are spatio-temporally extended and occur at multiple levels and at multiple time-slices in the language processing system. Any time lexical and other building blocks enter into the process of utterance interpretation or construction, and any time the linguistic input requires decomposition (presumably through analysis-by-synthesis) in order to contact the right lexical representations, Broca's region is recruited. The contentspecifics of the recruitment are determined by the specific regions and their connectivity profiles, at specific timeslices during processing. As is known for neurons in visual cortex [47], the real-time contribution of this region may well vary with time, as a consequence of the different dynamic cortical networks in which it is embedded at different points in time. This fits well with the finding that Broca's region as a whole is not language-specific, but also recruited in the service of other cognitive domains, such as music [48] and action [49], and with the finding that its contribution crosses the boundaries of semantics, syntax, and phonology [46]. Ideally, and in order to make progress, we need to determine both the function and the real-time contribution of Broca's region at time-slice t in the context of network $N(t)$.

\section{Beyond the classical model}

I have outlined the contours of a neurobiological model of language that deviates substantially from the classical WLG model, which was mainly based on lesion and single-word processing data. Three developments are worth highlighting: (i) the connectivity of the language cortex in left perisylvian regions is much more extended than proposed in the classical model and is not restricted to the arcuate fasciculus; (ii) the distribution of labor between the core regions in left perisylvian cortex is fundamentally different than proposed in the classical model. It assumes shared circuitry for core aspects of language production and comprehension, which both recruit temporal/parietal regions for retrieval of linguistic information that is laid down in memory during acquisition, and unification of building blocks into utterances or interpretations that are constructed on-line. Unification "enables words to cooperate to form new meanings" ([50]; p.179). (iii) The operation of language in its full glory requires a much more extended network than what is specified in the classical model. The basic principle of brain organization for higher cognitive functions is that these are based on the interaction between a number of neuronal circuits and brain regions that support the different contributing functional components. These circuits are not necessarily specialized for language, but nevertheless need to be recruited for the sake of successful language processing. One example is the attention network that might be triggered into operation by specific linguistic devices to safeguard against missing out on the most relevant (new, focused) information in the language input. The other example is the ToM network that seems crucial for designing our utterances with knowledge of the listener in mind and, as a listener, to make the step from 
coded meaning to speaker meaning (communicative intent). In addition, the role of the basal ganglia and the fronto-striatal loops in the overarching neurobiological infrastructure for language should not be neglected. Finally, the specific contribution to information processing of any brain region is dependent on its current state as well as the input it receives at any given point in time, which itself depends on the current computational environment in which it is embedded $\left[5^{\bullet}, 51\right]$.

\section{Conflict of interest statement}

Nothing declared.

\section{Acknowledgements}

I would like to thank Evelina Fedorenko, Tecumseh Fitch, and Karl Magnus Petersson for helpful comments on an earlier version. Alexander Backus helped with the artwork. The contribution was supported by a Spinoza Prize awarded to the author, and the Language in Interaction grant from NWO.

\section{References and recommended reading}

Papers of particular interest, published within the period of review, have been highlighted as:

- of special interest

$\bullet$ of outstanding interest

1. Fitch WT: The Evolution of Language. Cambridge: Cambridge - University Press; 2010,

This book provides an excellent introduction to the different theories about the evolution of language.

2. Jackendoff R: Foundations of Language: Brain, Meaning,

•- Grammar, Evolution. Oxford, UK: Oxford University Press; 2002, . This book is an excellent overview of the linguistic phenomena that any neurobiological account of language must be able to accommodate. Next to an in-depth analysis of the linguistic knowledge that humans command, it specifies a number of challenges for neuroscience.

3. Von Humboldt W: On the dual. Nouv Rev German 1829, 1:378-381.

4. Hagoort P: On Broca, brain, and binding: a new framework. Trends Cogn Sci 2005, 9:416-423.

5. Hagoort P: MUC (Memory, Unification, Control) and beyond. - $\quad$ Front Psychol 2013, 4 (Article 416).

The author provides an account of the core components of the language network and their connectivity. In addition, it is shown that linguistic markers of information structure trigger the contribution of the attentional networks during language processing. Finally, evidence is presented that the Theory of Mind (ToM) network plays a central role for inferring what the speaker intends to achieve when uttering a linguistic expression.

6. Werning M, Hinzen W, Machery M (Eds): The Oxford Handbook of Compositionality. Oxford: Oxford University Press; 2012.

7. Levelt WJM: A History of Psycholinguistics: The Pre-Chomskyan -. Era. Oxford: Oxford University Press; 2013,

This book contains the to date most scholarly and authoritative history of the sciences of language, mind and brain. Among other things, it includes a detailed sketch on the neurological contributions and debates in the 19th and 20th century about the neural architecture of the human language faculty.

8. Moorman S, Gobes SM, Kuijpers M, Kerkhofs A, Zandbergen MA Bolhuis JJ: Human-like brain hemispheric dominance in birdsong learning. Proc Natl Acad Sci U S A 2012, 109:12782-12787.

9. Caramazza A, Zurif EB: Dissociation of algorithmic and heuristic processes in language comprehension: evidence from aphasia. Brain Lang 1976, 3:572-582.

10. Menenti L, Gierhan SME, Segaert K, Hagoort P: Shared language: overlap and segregation of the neuronal infrastructure for speaking and listening revealed by functional MRI. Psychol Sci 2011, 22:1173-1182.
11. Segaert K, Menenti L, Weber K, Petersson KM, Hagoort P: Shared - $\quad$ syntax in language production and language comprehensionan FMRI study. Cerebral Cortex 2012, 22:1662-1670.

An $\mathrm{fMRI}$ study is presented in which repetition suppression is reported when sentences that consist of different words share their syntactic structure (e.g. passive, actives). This repetition suppression effect is observed for both within-modality (speaking-speaking; listening-listening) and between-modality (speaking-listening; listening-speaking) repetitions of syntactic structure. These findings provide evidence that the neural infrastructure of syntactic processing is shared between language production and language comprehension.

12. Friederici AD: Towards a neural basis of auditory sentence processing. Trends Cogn Sci 2002, 6:78-84.

13. Friederici AD: The cortical language circuit: from auditory

- $\quad$ perception to sentence comprehension. Trends Cogn Sci 2012, 16:262-268.

The author presents an overarching view of the different pathways involved in language processing and how during auditory sentence processing the information flow is orchestrated, starting from primary auditory cortex via ventral and dorsal routes to inferior frontal cortex. The ventral route is especially relevant for semantic processing, whereas the dorsal route is claimed to have a special role in the assignment of grammatical relations.

14. Hickok G, Poeppel D: The cortical organization of speech processing. Nat Rev Neurosci 2007, 8:393-402.

15. Poeppel D: Neuroanatomic and neurophysiological infrastructure for speech and language. Curr Opin Neurobiol 2014. this issue.

16. Pulvermuller F: How neurons make meaning: brain mechanisms for embodied and abstract-symbolic semantics. Trends Cogn Sci 2013, 17:458-470.

17. Hagoort P: How the brain solves the binding problem for language: a neurocomputational model of syntactic processing. Neuroimage 2003, 20:S18-S29.

18. Hagoort P: The fractionation of spoken language understanding by measuring electrical and magnetic brain signals. In The Perception of Speech. Edited by Moore BCJ, Tyler

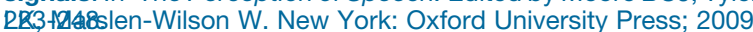

19. Binder JR, Desai RH: The neurobiology of semantic memory. - Trends Cogn Sci 2011, 15:527-536.

This paper presents a distributed view on the representation of concepts in semantic memory. Modality-specific sensory, action, and emotion systems provide input to high-level temporal and parietal convergence zones. These zones store increasingly abstract representations of entity (anterior temporal) and event knowledge (angular gyrus).

20. Hagoort $P$, Indefrey $P$ : The neurobiology of language beyond - $\quad$ single words. Annu Rev Neurosci 2014, 37.

A meta-analysis is presented of 151 imaging studies on syntactic and semantic unification. The meta-analysis reveals a clear dorsal/ventral gradient in both left inferior frontal cortex and left posterior temporal cortex, with dorsal foci for syntactic processing and ventral foci for semantic processing

21. Duncan J: How Intelligence Happens. New Haven and London: Yale University Press; 2010,

22. Baron SG, Osherson D: Evidence for conceptual combination in the left anterior temporal lobe. Neurolmage 2011, 55:1847-1852.

23. Price CJ: The anatomy of language: a review of $100 \mathrm{fMRI}$ studies published in 2009. Ann N Y Acad Sci 2010, 1191:62-88.

24. Fedorenko E, Thompson-Schill SL: Reworking the language - network. Trends Cogn Sci 2014, 18:120-126.

The authors propose a network perspective for understanding the neurocognitive architecture of language. They suggest that a language network consists of specialized core regions, together with a domain-general periphery. The analysis of core and periphery, they argue, should not be done on the basis of Regions of Interest, but instead on the basis of Networks of Interest (NOI). To account for language processing, one needs to investigate the dynamic interactions between the core and the periphery.

25. Mesulam M-M: From sensation to cognition. Brain 1998, 121:1013-1052.

26. Mclntosh AR: Large-scale network dynamics in neurocognitive function. In Coordination: Neural, Behavioral and Social 
Dynamics. Edited by Fuchs A, Jirsa VK. Berlin, Heidelberg: Springer; 2008:183-204.

27. Sporns O: Networks of the Brain. Cambridge, MA: MIT Press; 2011, .

28. Turken AU, Dronkers NF: The neural architecture of the language comprehension network: converging evidence from lesion and connectivity analyses. Front Syst Neurosci 2011, 5.

29. Johansen-Berg $\mathrm{H}$ : Human connectomics - What will the future demand? Neurolmage 2013, 80:541-544.

30. Uylings HBM, Malofeeva LI, Bogolepova IN, Amunts K, Zilles K: Broca's language area from a neuroanatomical and developmental perspective. In Neurocognition of Language. Edited by Brown CM, Hagoort P. Oxford: Oxford University Press 1999:319-336.

31. Amunts K, Zilles K: Architecture and organizational principles of Broca's region. Trends Cogn Sci 2012, 16:418-426.

32. Amunts K, Schleicher A, Ditterich A, Zilles K: Broca's region: cytoarchitectonic asymmetry and developmental changes. $J$ Compar Neurol 2003, 465:72-90.

33. Amunts K, Lenzen M, Friederici AD, Schleicher A, Morosan P, Palomero-Gallagher N, Zilles K: Broca's region: novel organizational principles and multiple receptor mapping. PLOS Biol 2010, 8

34. Amunts K, Catani M: Cytoarchitectonics, receptor architectonics, and network topology of language. In The Cognitive Neurosciences, edn 5. Edited by Gazzaniga MS. Cambridge, MA: MIT Press; 2015.

35. Xiang H, Fonteijn HM, Norris DG, Hagoort P: Topographical functional connectivity pattern in the perisylvian language networks. Cerebral Cortex 2010, 20:549-560.

36. Thompson-Schill SL, Bedny M, Goldberg RF: The frontal lobes - and the regulation of mental activity. Curr Opin Neurobiol 2005, 15:219-224.

In this paper the authors summarize the evidence against specialized modules of language in frontal cortex. They sketch the alternative view and evidence in favour of the claim that the frontal lobes might subserve general regulatory mechanisms across multiple domains (e.g. working memory, language comprehension).

37. Thompson-Schill SL: Dissecting the language organ: A new look at the role of Broca's area in language processing. In Twenty-First Century Psycholinguistics: Four Cornerstones. Edited by Cutler A. Lawrence Erlbaum Associates; 2005:173-190.

38. Berwick RC, Friederici AD, Chomsky N, Bolhuis JJ: Evolution, brain, and the nature of language. Trends Cogn Sci 2013, 17:89-98.
39. Friederici AD, Bahlmann J, Heim S, Schubotz RI, Anwander A: The brain differentiates human and non-human grammars: functional localization and structural connectivity. Proc Nat Acad Sci U S A 2006, 103:2458-2463.

40. Musso M, Moro A, Glauche V, Rijntjes M, Reichenbach J, Buchel C, Weiller C: Broca's area and the language instinct. Nat Neurosci 2003, 6.

41. Grodzinsky Y, Santi A: The battle for Broca's region. Trends Cogn Sci 2008, 12:474-480.

42. Fedorenko E, Duncan J, Kanwisher N: Language-selective and domain-general regions lie side by side within Broca's area. Curr Biol 2012, 22:2059-2062.

43. Tyler LK, Marslen-Wilson WD, Randall B, Wright P, Devereux BJ,

- Zhuang J, Stamatakis EA: Left inferior frontal cortex and syntax function, structure and behaviour in left-hemisphere damaged patients. Brain 2011, 134:415-431.

The authors report a study combining lesion analyses, functional imaging data, and behavioural data on syntactic processing. In controls, syntactic processing co-activated BA 45/47 in the left hemisphere and the posterior middle temporal gyrus. Voxel-based correlational analyses showed that tissue integrity and neural activity in BA 45 and left pMTG were correlated with preserved syntactic performance. It is this fronto-temporal network that needs to be intact for unimpaired processing of syntax.

44. Bozic M, Tyler LK, Ives DT, Randall B, Marslen-Wilson WD: Bihemispheric foundations for human speech comprehension. Proc Natl Acad Sci U S A 2010, 107:17439-17444.

45. Sahin NT, Pinker S, Cash SS, Schomer D, Halgren E: Sequential processing of lexical, grammatical, and phonological information within Broca's area. Science 2009, 326:445-449.

46. Hagoort P, Levelt WJM: The speaking brain. Science 2009, 326:372-373.

47. Lamme VA, Roelfsema PR: The distinct modes of vision offered by feedforward and recurrent processing. Trends Neurosci 2000, 23:571-579.

48. Patel AD: Language, music, syntax and the brain. Nat Neurosci 2003, 6:674-681.

49. Hamzei F, Rijntjes M, Dettmers C, Glauche V, Weiller C, Buchel C: The human action recognition system and its relationship to Broca's area: an fMRI study. Neuroimage 2003, 19:637-644.

50. Nowak M: Super Cooperators: Beyond the Survival of the Fittest. Why Cooperation, Not Competition is the Key to Life. EdinburghLondon: Canongate; 2011, .

51. Petersson KM, Hagoort P: The neurobiology of syntax: beyond string-sets. Philos Trans R Soc Lond B Biol Sci 2012, 3671971-1883. 